Annales Geophysicae (2002) 20: 1725-1735 (c) European Geosciences Union 2002

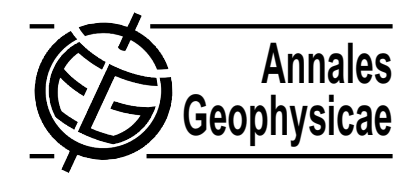

\title{
Polar observations of electron density distribution in the Earth's magnetosphere. 2. Density profiles
}

\author{
H. Laakso ${ }^{1}$, R. Pfaff ${ }^{2}$, and P. Janhunen ${ }^{3}$ \\ ${ }^{1}$ ESA Space Science Department, Noordwijk, Netherlands \\ ${ }^{2}$ NASA Goddard Space Flight Center, Code 696, Greenbelt, MD, USA \\ ${ }^{3}$ Finnish Meteorological Institute, Geophysics Research, Helsinki, Sweden
}

Received: 3 September 2001 - Revised: 3 July 2002 - Accepted: 10 July 2002

\begin{abstract}
Using spacecraft potential measurements of the Polar electric field experiment, we investigate electron density variations of key plasma regions within the magnetosphere, including the polar cap, cusp, trough, plasmapause, and auroral zone. The statistical results were presented in the first part of this study, and the present paper reports detailed structures revealed by individual satellite passes. The highaltitude ( $>3 R_{E}$ ) polar cap is generally one of the most tenuous regions in the magnetosphere, but surprisingly, the polar cap boundary does not appear as a steep density decline. At low altitudes $\left(1 R_{E}\right)$ in summer, the polar densities are very high, several $100 \mathrm{~cm}^{-3}$, and interestingly, the density peaks at the central polar cap. On the noonside of the polar cap, the cusp appears as a dense, $1-3^{\circ}$ wide region. A typical cusp density above $4 R_{E}$ distance is between several $10 \mathrm{~cm}^{-3}$ and a few $100 \mathrm{~cm}^{-3}$. On some occasions the cusp is crossed multiple times in a single pass, simultaneously with the occurrence of IMF excursions, as the cusp can instantly shift its position under varying solar wind conditions, similar to the magnetopause. On the nightside, the auroral zone is not always detected as a simple density cavity. Cavities are observed but their locations, strengths, and sizes vary. Also, the electric field perturbations do not necessarily overlap with the cavities: there are cavities with no field disturbances, as well as electric field disturbances observed with no clear cavitation. In the inner magnetosphere, the density distributions clearly show that the plasmapause and trough densities are well correlated with geomagnetic activity. Data from individual orbits near noon and midnight demonstrate that at the beginning of geomagnetic disturbances, the retreat speed of the plasmapause can be one L-shell per hour, while during quiet intervals the plasmapause can expand anti-earthward at the same speed. For the trough region, it is found that the density tends to be an order of magnitude higher on the dayside $\left(\sim 1 \mathrm{~cm}^{-3}\right)$ than on the nightside $\left(\sim 0.1-1 \mathrm{~cm}^{-3}\right)$, particularly during low $K_{p}$.
\end{abstract}

Key words. Magnetospheric physics (auroral phenomena;

Correspondence to: H. Laakso (hlaakso@so.estec.esa.nl) plasmasphere; polar cap phenomena)

\section{Introduction}

The modeling of the plasma number density in the magnetosphere is a difficult task for a number of reasons. First, the total density can significantly change on short temporal and spatial scales as a result of solar wind - magnetosphere - ionosphere coupling processes. In addition, it is difficult to gather reliable measurements of the total density in a tenuous environment, and hence, there are only limited amounts of density data reported to date from the Earth's vast magnetosphere.

Despite these difficulties, numerous studies of the electron density distribution within the magnetosphere have been reported previously. For example, Park et al. (1978), using the whistler wave technique, and Carpenter and Anderson (1992), using in situ measurements, studied the plasma density within the the plasmasphere. Besides modeling the density inside the plasmasphere, such data sets have also enabled models of the location of the plasmapause to be established (Gallagher et al., 1995).

Beyond the plasmapause, the electron density distribution becomes increasingly complex, especially during enhanced geomagnetic activity. For example, high convection velocities can rapidly change the distribution of the plasma density. Carpenter and Anderson (1992) modeled the plasma trough using ISEE data, and Gallagher et al. (1998) introduced a similar model as a function of $K_{p}$, using GEOS 2 observations of Higel and Wu (1984). Recently, Gallagher et al. (2000) presented a global plasma model for the entire inner magnetosphere. In general, however, the modeling efforts are impeded by a lack of comprehensive high-altitude data sets.

At higher latitudes, the modeling of the plasma density becomes even more complicated due to strong variations on short time scales. Also, the measurements available are quite sparse above $4 R_{E}$ distances, preventing empirical modeling 


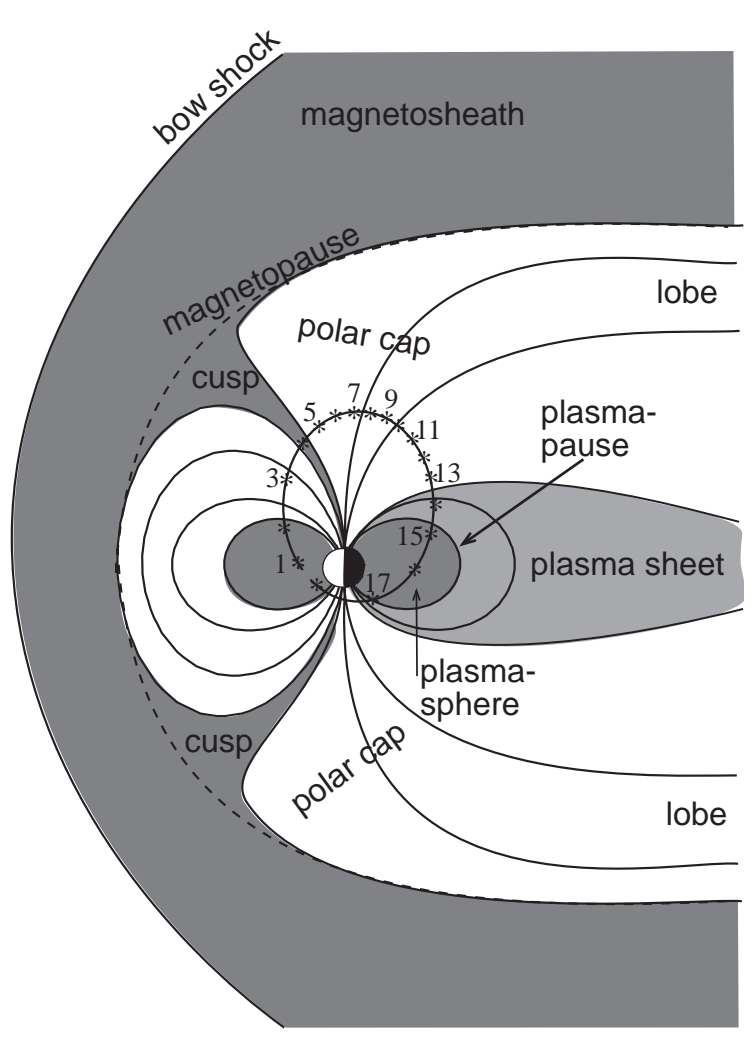

Fig. 1. Schematic drawing of the Earth's magnetosphere in the noon-midnight meridian. A solid line presents a Polar trajectory; asterisks give the spacecraft's position at one-hour intervals.

attempts. Persoon et al. (1983) used DE-1 observations to model the density distribution in the polar cap region between a 2 and $4.6 R_{E}$ distance. Using the same database, Persoon et al. (1988) investigated the formation of auroral cavities and showed that within these structures, some of the lowest densities within the magnetosphere may be found.

In the first part of this study, Laakso et al. (2002) investigated how the electron density varies statistically in the magnetosphere, using 45 months of satellite potential data from the EFI experiment of the Polar satellite. These data cover $10^{7}$ data points (one-minute averages) distributed between 2 and $9 R_{E}$ geocentric distances along Polar's polar orbit. Using high-resolution data from individual orbits, the present paper investigates several plasma regions that have distinct density signatures, such as the polar cap, cusp, trough, plasmapause, and auroral zone. In particular, the observations are used to show how these regions evolve with time and geomagnetic activity.

\section{Density variations in the specific magnetospheric regions}

We use measurements gathered by the electric field instrument (EFI) on the Polar satellite. Polar was launched on 24 February 1996, into a $90^{\circ}$ inclination orbit with a $9 R_{E}$ geocentric apogee distance (initially over the Northern Hemi- sphere), a $1.8 R_{E}$ perigee distance (initially over the Southern Hemisphere), and an orbital period of about $18 \mathrm{~h}$. The orbital plane rotates about the Earth with respect to the Sun in one year so that all local times are covered in a 6-month period. Figure 1 presents a schematic drawing of the magnetosphere at the noon-midnight meridian and a Polar orbit, where asterisks mark the position of the satellite at one-hour intervals.

This section presents plasma density profiles in selected regions that are frequently encountered by Polar, such as the polar cap, cusp, auroral zone, trough, and plasmapause. Figure 2 shows the electron densities derived from spacecraft potential data gathered with the electric field instrument for seven consecutive orbits near the noon-midnight meridian on 29 April to 4 May 1996. Each panel displays measurements between subsequent dayside equatorial crossings (see orbit in Fig. 1). In each of the panels in this presentation, the spacecraft is first in the high-density plasmasphere, where the density is typically several $100 \mathrm{~cm}^{-3}$ or more, and is moving towards the northern polar cap in the noon sector.

After about an hour, the density decreases a few orders of magnitude when the satellite completes its outbound crossing of the plasmapause (PP). The approximate locations of the plasmapause for the top panel are marked with arrows. A few hours later, a significant, large-scale density enhancement is observed when Polar enters the northern cusp. Poleward of this region, the density becomes very low as the satellite encounters the polar cap. Polar subsequently moves into the night sector, and at the equatorward edge of the polar cap, it crosses the auroral zone and later the plasmasphere. At the end of each interval, low densities are detected when the satellite passes through the auroral zone and polar cap over the Southern Hemisphere. These encounters are quite short during this portion of the orbit due to the low altitude and high speed of the satellite.

Although the density variations are quite similar from orbit to orbit, there are numerous differences between orbits which reveal fascinating and complex features, such as large-scale density patterns in the polar cap, the structure of the cusp, density cavitation at the auroral zone, characteristics of the density decline at the plasmapause and so on. Their structure and dynamics are revealed to the fullest extent when the data are studied with high time resolution, with respect to invariant variables, such as L-shell and invariant latitude.

\subsection{Polar cap}

The statistical study by Laakso et al. (2002), as well as the data shown in Fig. 2, imply that the electron density is very low in the high-altitude polar cap region. The polar cap density strongly depends on season (solar illumination), geomagnetic activity, and altitude. Next, we proceed with our investigation by studying some individual passes through the polar cap region. As the spatial density variation in the polar cap's noon-midnight meridional plane is strongly influenced by the positions of the cusp and auroral zone, we use po- 
lar cap data from the dawn-dusk meridian for this particular study.

Figure 3 presents the polar cap density measurements from 1-6 January 1997, versus invariant latitude for both northern winter (at high altitudes, red points) and southern summer (at low altitudes, blue points) conditions. The left panels are for the dawn sector and the right panels for the dusk sector. The bottom panels show the spacecraft distance for the Southern and Northern Hemisphere passes. If the derived density exceeded $300 \mathrm{~cm}^{-3}$, it was given that value. Recall that our method for deriving the plasma density becomes increasingly unreliable for high electron fluxes (Laakso et al., 2002). Such measurements are useful, nevertheless, as indicators of how often high-density plasmas are encountered.

When interpreting the polar cap measurements of Fig. 3, it is important to bear in mind that the northern polar cap is in a shadow for most of the time, whereas the Sun is continuously ionizing the southern polar cap. For comparison, Fig. 4 presents measurements at the same meridian six months later, on 5-10 July 1997, during the northern summer and the southern winter where these conditions are reversed.

In the high-altitude ( $>4 R_{E}$ ) winter polar cap (see Fig. 3), the data points are concentrated primarily in the range of $0.01-1 \mathrm{~cm}^{-3}$. The equatorward boundary of the polar cap does not appear very clearly. On the dawnside, the data suggest the polar cap boundary may be near $73-75^{\circ}$, whereas on the duskside a steep decline occurs near $71^{\circ}$. In summer (see Fig. 4), the high-altitude polar cap density, particularly on the dawnside, is more variable and somewhat higher, ranging between 0.05 and $10 \mathrm{~cm}^{-3}$. On the dawnside, the polar cap boundary likely occurs near $76^{\circ}$, whereas on the duskside it possibly exists at $70^{\circ}$.

At low altitudes $\left(\sim 0.9 R_{E}\right)$, there is no distinct density decline at the polar cap boundary in either season. In winter (Fig. 4), the low-altitude polar cap density usually remains below $50 \mathrm{~cm}^{-3}$, the densities are highly variable, with values below $1 \mathrm{~cm}^{-3}$ frequently observed. In summer (Fig. 3), the densities are significantly higher, seldom below $10 \mathrm{~cm}^{-3}$ and often above $100 \mathrm{~cm}^{-3}$. Furthermore, interestingly, the density is quite constant and higher in the polar cap than at lower latitudes; notice that lower densities above $85^{\circ}$ are mainly due to orbital coverage. The dawn-dusk asymmetry may well be associated with the IMF orientation and the structure of the convection shell. The investigation of such details are however, beyond this study.

\subsection{Cusp}

The cusp is the region where the solar wind plasma has direct access to the Earth's environment (Smith and Lockwood, 1996). In the magnetosheath, the average solar wind density is $50-100 \mathrm{~cm}^{-3}$, which is an order of magnitude higher than the solar wind density and two orders of magnitude higher than densities in the outer regions of the dayside magnetosphere. Thus, the density should be relatively high in the cusp. In fact, this is exactly how the cusp is distinguished in Fig. 2, i.e. as a local enhancement of the plasma density,
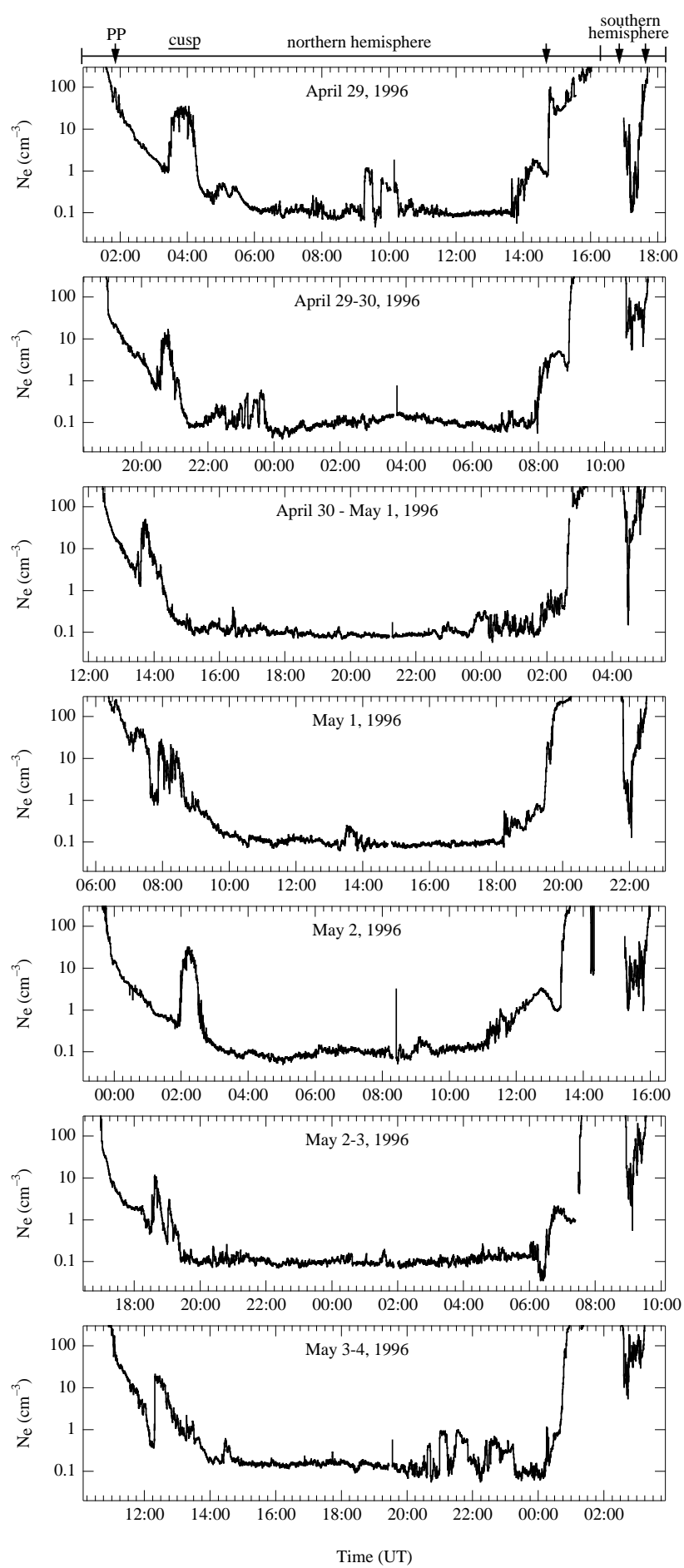

Fig. 2. Electron density variation on eight consecutive orbits of 29 April to 4 May 1996. The satellite's orbital plane is near the noonmidnight meridian. Each panel presents a full orbit of data, from one perigee to the next one.

when the satellite moves from the dayside magnetosphere through the cusp into the polar cap (Marklund et al., 1990; Palmroth et al., 2001; Laakso et al., 2002).

As it appears in Fig. 2, both the cusp position and plasma 

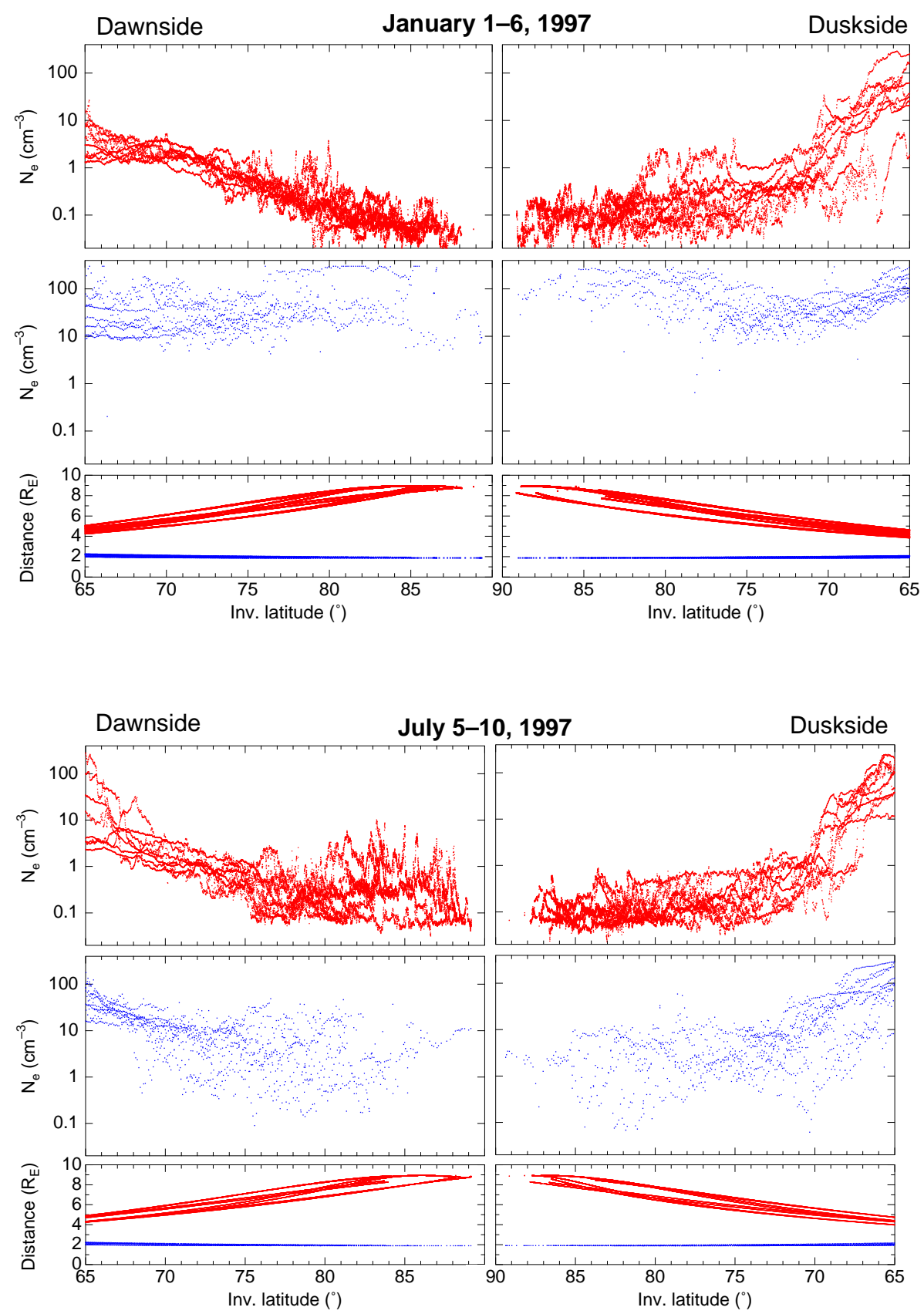

Fig. 3. Polar cap density plotted against invariant latitude for 1-6 January 1997. The panels from top to bottom are: electron density in the high-altitude northern polar cap, electron density in the low-altitude southern polar cap, and the altitudes of the satellite during the polar cap observations. The red color is used for the northern polar cap and the blue color for the southern polar cap. Here the northern polar ionosphere is in darkness and the southern polar ionosphere is sunlit.
Fig. 4. Same as Fig. 3 but for the interval 5-10 July 1997. Contrary to Fig. 3, here the northern polar ionosphere is illuminated by the Sun and the southern polar ionosphere is in darkness. density are highly variable. To illustrate this further, Fig. 5 displays the electron density versus invariant latitude for the cusp crossings in Fig. 2. The thickness of the cusp in these examples varies between $1-3^{\circ}$ in invariant latitude, which is more than that observed with low-altitude DMSP satellites (Newell and Meng, 1992).

Both the location and density of the cusp can significantly change from orbit to orbit. Although high-altitude cusp crossings by Polar may last between 0.5-4h (Palmroth et al., 2001), depending on the satellite's altitude, in Fig. 5 the crossings last only $0.5-1 \mathrm{~h}$. In the cusp, the average density is usually several $10 \mathrm{~cm}^{-3}$ and can sometimes exceed $100 \mathrm{~cm}^{-3}$, although not in the cases shown in Fig. 5. Note that such densities are of the order of the magnetosheath density and typically an order of magnitude higher (or more) than in the magnetospheric regions adjacent to the cusp.

Since Polar may spend considerable time crossing the cusp region, the cusp itself may evolve and change dynamically during this time. In the fourth panel from the top in Fig. 5, for example, it seems that the cusp is encountered twice. We have examined the IMF observations from the IMP-8 and Wind satellites during this event and found that simultaneously with this crossing, the IMF orientation had two large excursions. In fact, Polar has encountered several other events like this, which all suggest that the cusp position is very sensitive to the IMF orientation, and the cusp position 
can more or less instantaneously be influenced by IMF variations.

Another common feature in the cusp density profiles is a steep density gradient on the equatorward boundary of the cusp, which is caused by a rapid motion of the cusp over the satellite rather than the satellite's movement over a stationary cusp (the satellite's speed is only a few $\mathrm{km} \mathrm{s}^{-1}$ ). Figure 5 shows three exceptions from this common feature, namely the examples in panels 2, 5 and 8 from the top, where the density profiles are quite symmetric, which suggests that the cusp was stationary during those crossings.

\subsection{Plasma trough}

The average densities presented by Laakso et al. (2002) demonstrate that the densities tend to be higher on the dayside than on the nightside. In particular, the average densities between the plasmapause and the magnetopause on the dayside can be quite high, more than $1 \mathrm{~cm}^{-3}$, whereas on the same L-shells at the dusk, dawn, and midnight sectors, the average densities appear to be lower. To investigate this in more detail, we study eight consecutive orbits in the noonmidnight meridian on 25 April to 1 May 1998. Figure 6 displays electron density against L-shell for midnight (dotted lines) and noon (solid lines). The date and UT time in each panel refer to the observations on the nightside; the dayside measurements were collected 3-4h later. Each panel also gives a $K_{p}$ range for the measurement interval, including a six-hour interval prior to the measurements.

The top two panels of Fig. 6 are taken during an active period, and no significant differences between nightside and dayside can be detected, except that the nightside profiles are more variable. When the geomagnetic activity is lower (all the other panels), the dayside density tends to be higher than the nightside one, often by an order of magnitude. In the second panel from the bottom, a storm sudden commencement (SSC) occurs between the nightside and dayside observations, which perturbs the density structure around the Earth. The evolution of the plasmapause location shows some interesting features that are considered in the next section.

In conclusion, for active intervals the plasma density is of the same order of magnitude between the dayside and the nightside, but the nightside densities are more variable. For quiet intervals, the dayside densities are clearly higher than the nightside ones.

\subsection{Plasmapause}

Above the ionosphere's peak daytime density of $\sim 10^{6} \mathrm{~cm}^{-3}$ at about $300 \mathrm{~km}$ altitude, the density decreases exponentially with altitude out to the plasmapause, which is usually located in the $L=3-7$ range, depending on magnetic activity. During extremely quiet conditions, the plasmapause can move beyond $L=10$ on the dayside (Laakso and Jarva, 2001). At the plasmapause, the density rapidly declines by a few orders of magnitude over a relatively short distance (see, e.g. Carpenter and Anderson, 1992). Near this location the plasma
POLAR EFI experiment

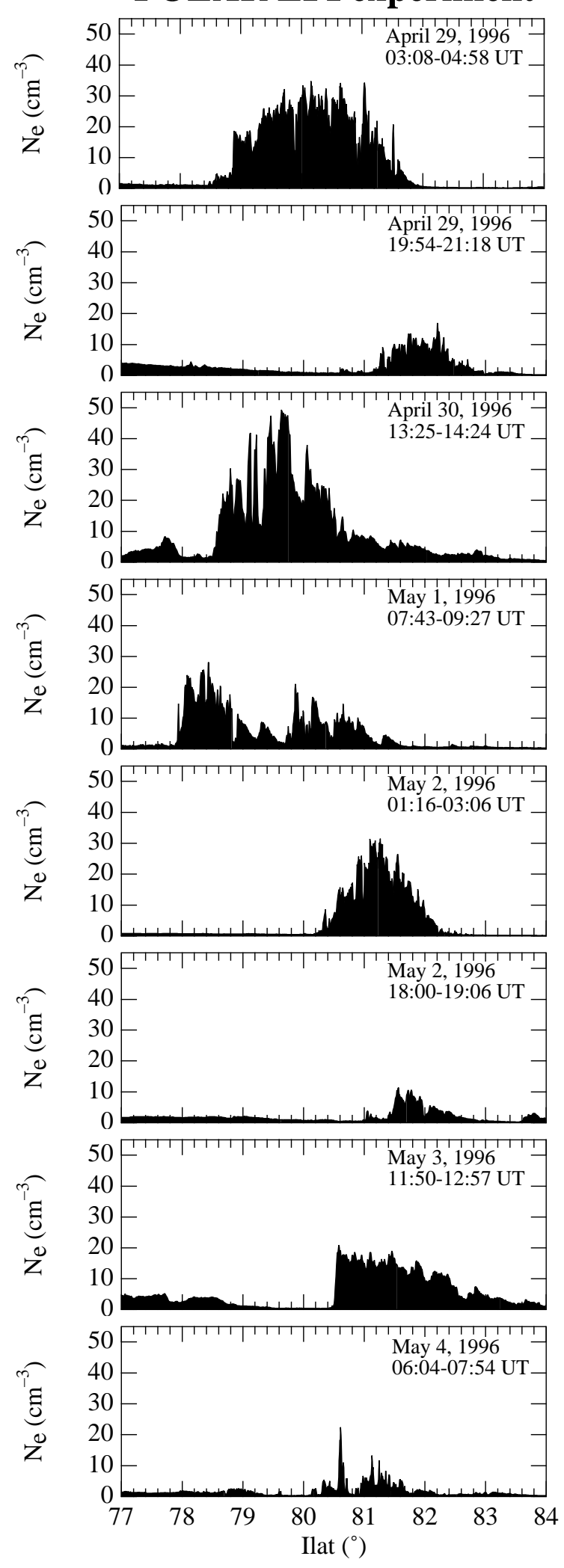

Fig. 5. Electron density is plotted against invariant latitude for the Northern Hemisphere cusp crossings of Fig. 2. 

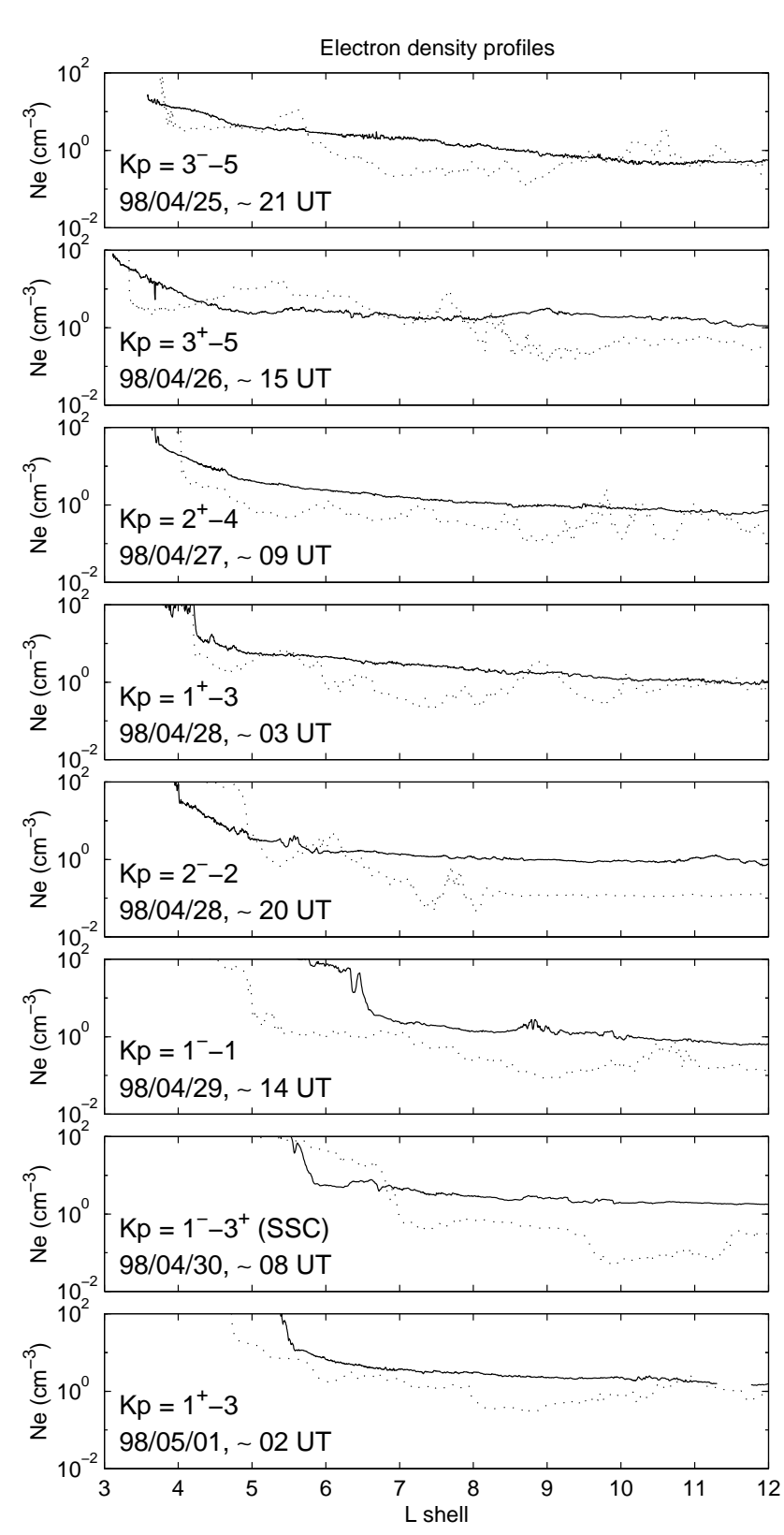

Fig. 6. Electron density plotted against L-shell for midnight (dotted lines) and noon (solid lines); the date and UT time refers to the nightside observations, the dayside data are taken 3-4 h later.

drift is switched from corotation into convection, which is called a flow separatrix (Lyons and Williams, 1984). During stationary conditions, the two boundaries happen at the same distance, but for variable conditions, this is not the case (Lemaire and Gringauz, 1998). In general, the characteristics of the plasmapause can vary quite significantly, depending on local time, altitude, and geomagnetic activity (Moldwin et al., 1994; Gallagher et al., 1995; Elphic et al., 1996).

When the plasmapause appears at low L-shells, the density decline occurs near $100 \mathrm{~cm}^{-3}$, but as the boundary moves away from the Earth, the steep decline happens in a lower density range. This is weakly visible in Fig. 6 when the plasmapause occurs at around $10 \mathrm{~cm}^{-3}$ in the bottom three panels and in a higher density range in the top panels. In addition, the value of the plasma density near the plasmapause is essentially dependent on the refilling rates of the plasmasphere during the preceeding few days (Lawrence et al., 1999).

The Polar satellite crosses the plasmapause usually four times per orbit, providing an excellent opportunity to study the structure and evolution of the plasmapause. Over the Northern Hemisphere, the plasmapause crossing occurs at the 2-5 $R_{E}$ altitudes near the magnetic equator, while over the Southern Hemisphere it happens at roughly $1 R_{E}$ altitude at high magnetic latitudes $\left(<-40^{\circ}\right)$. Each panel in Fig. 2 contains a plasmapause crossing over the Northern Hemisphere near noon in an hour after the start of the interval and near midnight some $3-4 \mathrm{~h}$ before the end of the interval. The last two plasmapause crossings occur within $1-2 \mathrm{~h}$ over the Southern Hemisphere.

The local time asymmetry of the plasmapause is an important issue. It is not easy to solve this problem, as it requires simultaneous observations on both sides of the Earth, or very stationary conditions. At the noon-midnight meridian the asymmetry is expected to be quite weak. However, in Fig. 6, the dayside and nightside plasmapause usually appear at different L-shells (except panel 4 from top), which shows that the evolution of the plasmapause can be quite fast, as the dayside and nightside observations are collected $3-4 \mathrm{~h}$ apart.

To investigate further the evolution of the plasmapause, we again turn to the geomagnetically variable interval on 22 April to 1 May 1998, when Polar crosses the plasmapause near noon (11:00-12:00 MLT) and midnight (23:0024:00 MLT). While the density profiles are displayed in Fig. 6, Fig. 7 shows the locations of the plasmapause every $18 \mathrm{~h}$ over the Northern Hemisphere. The variation of the $K_{p}$ index is shown by a dotted line. The scale on the left is for the plasmapause position and the scale on the right for the $K_{p}$ index. In the beginning of the interval, the geomagnetic activity is weak, and the plasmapause stays near $L=5$. On 23 April, a quiet interval occurs at 9:00-18:00 UT, during which the nightside plasmapause rapidly moves anti-earthward; the dayside evolution cannot be evaluated due to a lack of measurements. A magnetic storm on 23-27 April is preceeded by an SSC on 23 April, 18:25 UT. The dayside plasmapause is crossed 30 min later, and it seems plausible that the plasmapause has already moved at least $0.5 \mathrm{~L}$ earthward. Notice that we do not know the distance of the dayside plasmapause at the end of the quiet interval, but it is very likely that it was at least near the same distance where it was during the previous measurement due to the quiet period of 23 April.

During the storm on 24-25 April, the plasmapause appears near $L=3$. For the declining phase of magnetic activity, the plasmapause moves outward from the Earth both in the noon and midnight sectors. 29-30 April is a particularly quiet period, during which the plasmasphere expands rapidly. A fast earthward movement of the plasmapause occurs after an SSC onset at 09:28 UT on 30 April 1998. Polar crosses the dayside plasmapause three hours after the onset and by then 


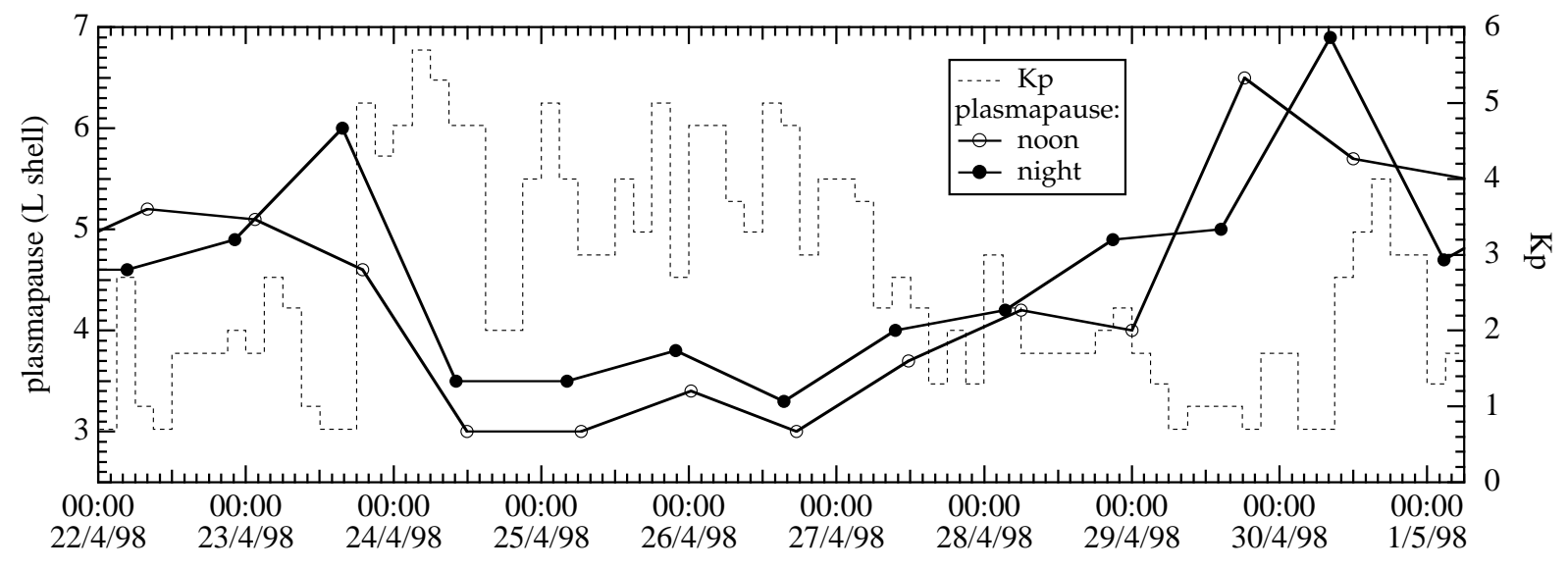

Time (UT)

Fig. 7. Locations of the plasmapause near noon and midnight on 22-30 April 1998; the L-shell scale from 3 to 7 is given on the left axis. The panel also presents the variation of the 3 -h global $K_{p}$ index, shown by a dashed line, with a scale on the right axis.

this boundary has retreated earthward by at least one L-shell. Once more, we do not know the plasmapause distance just before the onset, but again the preceeding quiet period has likely kept the plasmapause at the same position as during the previous crossing or preferably has moved it away from the Earth. Thus, we speculate that the plasmapause may be reacting quite rapidly to the changes in the convection electric fields. As a result, the location of the noon and midnight plasmapause keeps changing places in the last four panels of Fig. 6.

We emphasize that these measurements are made at the noon-midnight meridian, and the evolution can be significantly different at other local time sectors, especially in the dusk sector (see, e.g. Moldwin et al., 1994; Eplhic et al., 1996). We will discuss our findings further in Sect. 3.3.

\subsection{Auroral oval}

The auroral zone is one of the most tenuous regions within the magnetosphere. Particularly low densities can be observed in auroral cavities (Persoon et al., 1988; Makela et al., 1998). Persoon et al. (1988) found clear evidence that auroral cavitation is a result of auroral acceleration processes, and according to Makela et al. (1998), there is no correlation between the occurrence of cavities and geomagnetic activity.

On the nightside Polar crosses the auroral zone over the Southern and Northern Hemispheres with a separation of about $2-3 \mathrm{~h}$. A major difference between the observations from the two hemispheres is the observation distance, which is in the range of 3.5-5.5 $R_{E}$ for the Northern Hemisphere and about $1.9 R_{E}$ for the Southern Hemisphere. Due to the compressed time scale in Fig. 2, density variations above the auroral oval are not clear, except that enhanced disturbances with some density cavities occur.

We continue our analysis by examining seven consecutive auroral oval crossings on 25-30 April 1998, which is an interval of highly variable activity. Figures $8 \mathrm{a}$ and $\mathrm{b}$ present the density profiles against invariant latitude: Fig. $8 \mathrm{a}$ is for the Northern Hemisphere, and Fig. 8b is for the Southern Hemisphere. Notice that the density scales are different for these figures. The magnetic local time of the observations is given in each panel. Using a preliminary global $A E$ index, we have defined the level of global magnetic activity for each crossing, as indicated in the panels. These examples present density profiles collected during all substorm phases and during very quiet periods.

Although the northern and southern oval crossings are separated by a few hours, in some panels one can distinguish similar features between the two profiles. On the other hand, the measurements in the two adjacent panels over one hemisphere are separated by about $18 \mathrm{~h}$. It is clearly obvious that these profiles are not correlated.

The density profiles across the auroral zone are highly variable and presumably quite low densities should be encountered. Therefore, it is somewhat surprising that it is not always easy to distinguish the auroral cavities in Figs. 8a and b. Note, however, that the occurrence of a low density region is not enough to call it a cavity, since at high latitudes such events may be caused by irregular transitions to the polar cap where low densities are also encountered. Therefore, we have investigated dc-electric field variations during these events, because the electric fields are usually larger and more variable in the auroral zone than in the polar cap. The locations of enhanced electric fields are marked with solid horizontal bars in the panels. These disturbances are usually accompanied by magnetic field perturbations. If particularly strong electric fields (i.e. more than $15 \mathrm{mV} \mathrm{m}^{-1}$ for the northern oval, and more than $45 \mathrm{mV} \mathrm{m}^{-1}$ for the southern oval) are detected, it is marked with dotted horizontal bars.

One can observe that clear density cavities in the auroral zone do not always exist. Another obvious fact is that auroral cavities and auroral electric fields do not fully overlap: there are cavities with no electric field signatures, and 


\section{POLAR EFI experiment}
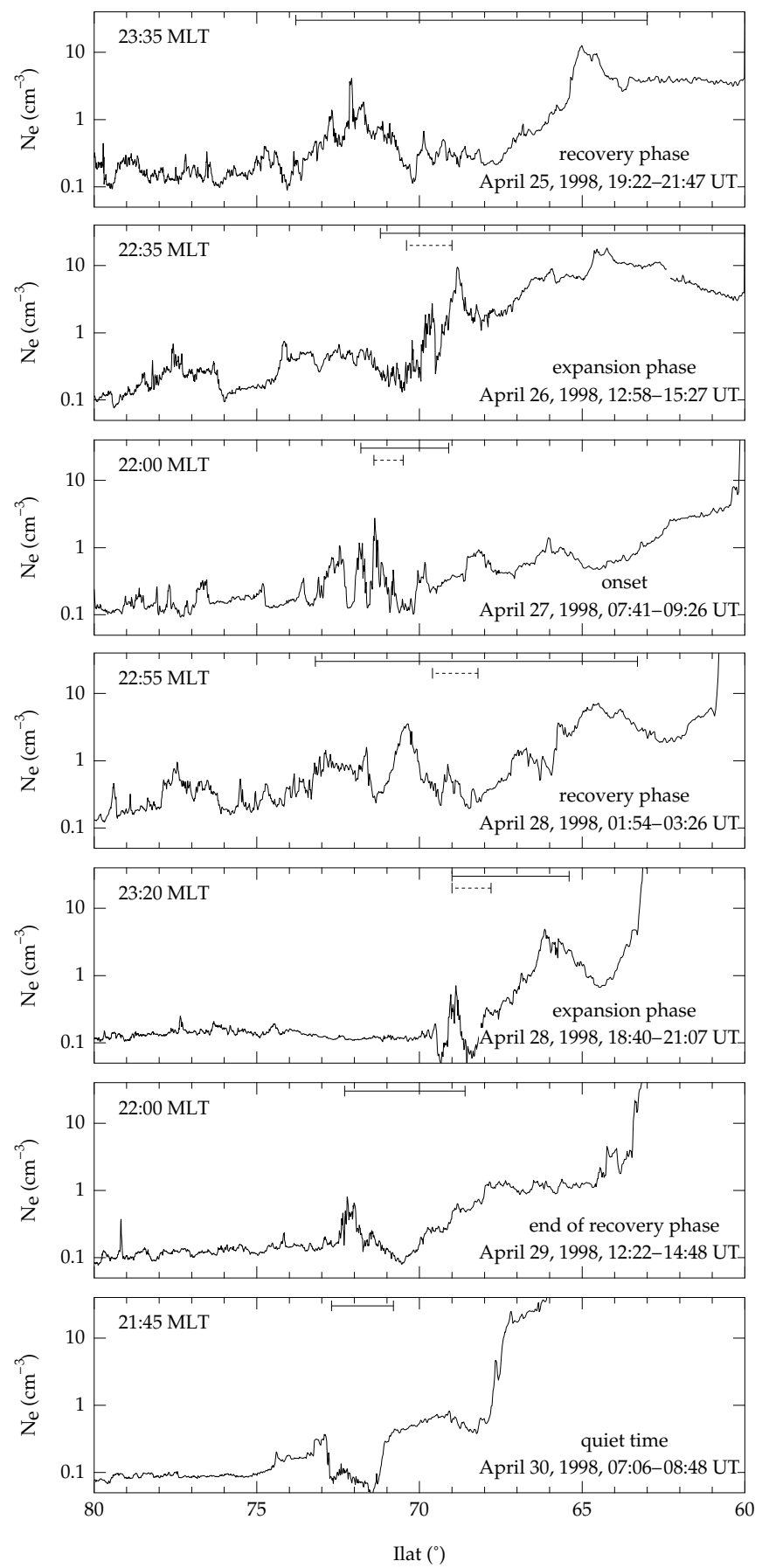

POLAR EFI experiment
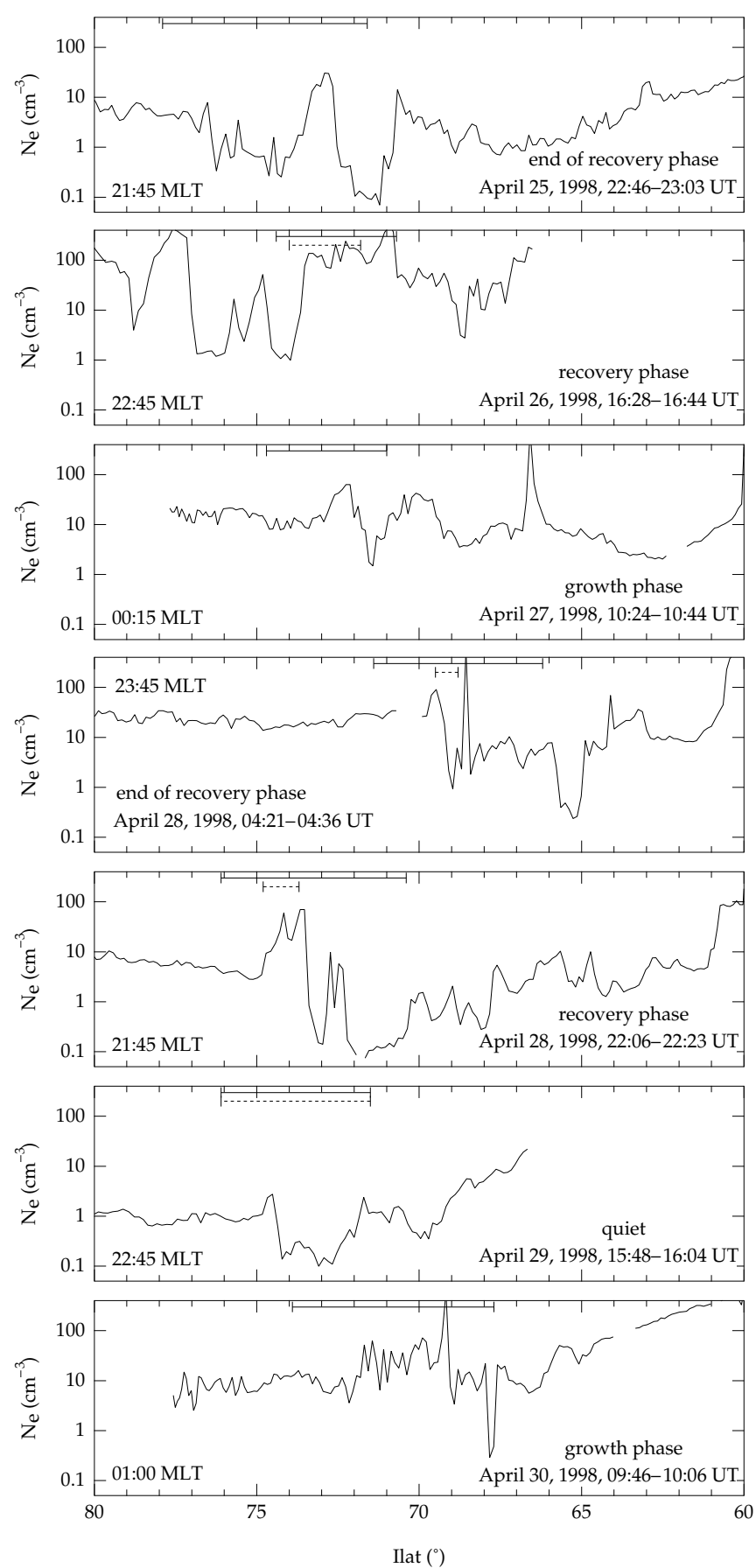

Fig. 8. (a) Crossings of the northern auroral zone in the pre-midnight sector on seven consecutive orbits of 25-30 April 1998. The crossings (the UT interval is given in each panel) happened during different geomagnetic conditions, as marked in each panel. The solid horizontal bars indicate the locations of the electric field perturbations; the dashed bars show the locations of very strong electric fields, when they existed. (b) same as (a) but for the southern auroral zone.

vice versa, there are large electric field perturbations with no clear density cavitation. This is a suprise to some extent, as the auroral electric fields tend to be anti-correlated with the level of plasma density (see, e.g. Lindqvist and Marklund, 1990). A clear case of an auroral cavity exists in the third plot from the bottom on 28 April (see both Figs. 8a and b). In this event, Polar crosses the auroral zone very soon after the onset of an isolated substorm. Two distinct cavities are observed over the Northern Hemisphere (Fig. 8a); however, electric field disturbances appear only within the inner 
cavity. One may wonder if the other one is due to a polar cap transition. This is not likely because two hours later, during the recovery phase of the same substorm, the same cavities are still observed, now over the southern oval (Fig. 8b), at slightly different invariant latitudes, possibly due to different MLT or of an evolution of the oval. In the next panel below, about $18 \mathrm{~h}$ later, Polar detects one wide cavity just after the end of the recovery phase of a moderate substorm. Two hours later, when the substorm is over, the cavity still exists above the southern oval. The bottom panel in Fig. 8a shows a deep and wide cavity during a very quite interval, some five hours after a substorm recovery phase. However, over the southern oval, no cavitation is observed, possibly due to the high altitude of the bottom of the cavity. In the top panels of Figs. 8a and $b$, one cannot easily distinguish any cavities. These observations tend to suggest that the formation and existence of cavitation at the auroral zone is not a straightforward problem.

\section{Discussion}

\subsection{Motion of the cusp}

According to the statistical results by Laakso et al. (2002), the cusp position is quite stationary for low $K_{p}$ and tends to move equatorward with increasing $K_{p}$ (for a detailed study, see Palmroth et al., 2001). During disturbed conditions, however, the cusp disappears in the average density picture, even though the cusp is always observed in the noon sector, except for some rare cases, such as the quiet period of 10-11 May 1999, when no cusp was observed. Our conclusion is that the cusp position can change quite dramatically under geomagnetically active intervals. We now discuss evidence for this from individual cusp crossings.

We have found several cases where the cusp is apparently crossed twice; one such event is shown in Fig. 5. The examination of the interplanetary magnetic fields from several spacecraft shows that these events occur simultaneously with variations of the IMF. This implies that the cusp can shift its position nearly instantly in response to an IMF variation. Another related feature is that individual cusp crossings frequently reveal steep density slopes at the equatorward boundary of the cusp. This is likely to be caused by a motion of the cusp, as the spacecraft speed is only a few $\mathrm{km} \mathrm{s}^{-1}$. When the solar wind is stationary, the cusp crossing can be described by the spacecraft moving slowly through a stationary cusp. Figure 5 contains examples of such cases in the second, fifth and eighth panels from the top. Notice that the density decline is usually not steep on the poleward boundary of the cusp, because the densities in the cusp and the plasma mantle are somewhat similar and the latter region is rather wide (Rosenbauer et al., 1975).

Berchem and Russell (1982) investigated the characteristics of the dayside magnetopause and found that it is in constant irregular motion at velocities $10-80 \mathrm{~km} \mathrm{~s}^{-1}$. Since the cusp motion is not driven by magnetosheath pressure varia- tions but rather by reconnection at the magnetopause (Smith and Lockwood, 1996), it is likely to evolve at somewhat different velocities. Unfortunately with only one satellite we cannot properly solve the velocity of the boundary, but it seems often to be much higher than the velocity of the vehicle and thus, likely of the order of $10 \mathrm{~km} \mathrm{~s}^{-1}$ at high altitudes. Therefore, the cusp can assume a new position in less than $10 \mathrm{~min}$ in most cases. This suggests that an appropriate integration time of the solar wind measurements needed for studying the dynamics of the cusp is a few tens of minutes.

\subsection{Density in the trough}

We studied a sequence of orbits between $L=3-12$ and found that the density between the plasmapause and the magnetopause is higher on the dayside than on the nightside (see Fig. 6). This is particularly clear for quiet intervals, where the difference may be almost an order of magnitude, whereas during disturbed conditions, the densities tend to be roughly the same. One noticeable feature in the density profiles is that the dayside profiles do not change much (except the position and slope of the plasmapause), whereas the nightside densities can develop strongly from orbit to orbit. The explanation for the difference is apparently due to the ionosphere which represents a source of plasma for the dayside magnetosphere. This source is less important for the nightside magnetosphere. During quiet periods, the difference, therefore, reflects the ionospheric plasma contribution, by yielding different rates at noon and midnight, whereas during disturbed conditions the nightside near-Earth magnetosphere has additional sources, such as the plasmasphere via erosion processes, and the magnetotail via fast magnetospheric convection that transport plasmas from the tail toward the Earth. Another possible explanation for the higher densities on the dayside is that as flux tubes convect from the nightside towards the dayside, they are continually fed by ionospheric outflows at a rate of around 0.5 to $20 \mathrm{~cm}^{-3} /$ day (Lawrence et al., 1999).

By comparing the density profiles of Fig. 6 with the trough model of Carpenter and Anderson (1992), we found that the densities agreed within a factor of $2-5$ on the dayside. On the nightside we used the model at 00:00 MLT, and again, similar differences were obtained. These differences are close to within the experimental error (factor of 2-3), but in most cases the difference appears consistently in the same direction (i.e. our densities are usually lower than those of Carpenter and Anderson). One possible reason for the discrepancy may be that the Carpenter and Anderson model does not take into account the geomagnetic activity level, which has an impact on the trough density.

\subsection{Evolution of the plasmapause}

The coupling between the plasmasphere and the outer magnetosphere is not yet well understood (Moldwin, 1997). Erosion and recovery of the plasmasphere are important issues that are inherently related to the global dynamics and struc- 
ture of the magnetosphere. At the plasmapause, the density suddenly decreases a few orders of magnitude over a relatively short distance (see, e.g. Carpenter and Anderson, 1992), nearly coinciding with the switch of the plasma drift from corotation to convection (Lyons and Williams, 1984). Naturally, the location of flow separatrix can move to a new position rapidly, when the convection electric field, driven by the solar wind, changes, whereas the plasmapause, defined by the steep density decline, evolves at a slower speed, depending on the time scales of the refilling and erosion processes.

We investigated a sequence of plasmapause crossings by Polar at 18-h intervals near local midnight and noon (Fig. 7). The observations suggest that the plasmasphere can evolve faster than usually assumed. On 23 April 1998, the dayside plasmapause is crossed 30 min after an SSC onset, during which the plasmapause has apparently moved earthward more than $0.5 \mathrm{~L}$. Similarly after another SSC, on 30 April 1998, a speed of the same magnitude is detected. On the other hand, two quiet periods occurred during the same interval and they exhibited rapid expansions of the plasmasphere at about the same speed. These examples suggest that the time scales important to the evolution of the plasmasphere can be a few hours rather than 10-20 h (see, e.g. references in Gallagher et al., 1995). If the speed of the plasmapause evolution is one L-shell in 1-2 $\mathrm{h}$, the plasmapause can assume any distance relative to the flow separatrix in less than $5 \mathrm{~h}$. Once the plasmapause has assumed a new position, the refilling of the flux tubes takes a much longer time (Lawrence et al., 1999).

When interpreting Polar observations, one must bear in mind that the crossings occur at 18 -h intervals, and the plasmapause location can develop significantly within such a time, which makes the interpretation difficult. Also, the evolution at different local time sectors can be quite different. Particularly strong evolution is observed in the bulge region (Moldwin et al., 1994; Elphic et al., 1996). For instance, Elphic et al. (1996) have found that the plasmaspheric bulge actually moves sunward after SSC onsets. Our observations at both noon and midnight indicate clearly that the plasmapause moves earthward at these two sectors. The statistical pictures by Laakso et al. (2002) (see their Fig. 4) suggest that in the afternoon sector the average plasmapause/trough density remains unchanged for high $K_{p}$. This is possibly due to erosion processes that cause detached plasmaspheric fragments that drift sunward in the dusk sector, keeping the average density high.

\section{Summary}

Differential potential $(\Delta V)$ measurements from the Polar electric field instrument have been used to study electron density profiles in some specific regions of the magnetosphere between $2-9 R_{E}$ geocentric distances. This technique provides a measure of the thermal plasma density to values even below $0.1 \mathrm{~cm}^{-3}$ with high time resolution (of time scales less than $0.1 \mathrm{~s}$ ). The statistical results are presented by Laakso et al. (2002), and the present paper displays density profiles across some key plasma regions of the magnetosphere. The major findings are:

1. The polar cap is one of the most most tenuous regions within the magnetosphere. Its equatorward boundary, however, does not appear as a steep density decline because equally low densities can be detected in the auroral zone. The polar cap density data show clear asymmetry with MLT. In summer at low altitudes, the polar cap density is quite high in the central polar cap, that is, of the same order of magnitude as at lower latitudes, while this is not observed in winter or at high altitudes.

2. The cusp properties, such as the electron density and the location, change significantly from orbit to orbit. A typical density is between 40 and $100 \mathrm{~cm}^{-3}$ in the high-altitude (4-8 $R_{E}$ ) cusp. Based on individual cusp crossings, we found several cases where the cusp can assume a new position, responding almost instantaneously to changes in the IMF orientation.

3. The plasmapause location is strongly correlated with the $K_{p}$ index so that its asymmetry in MLT increases with $K_{p}$. The strongest evolution in the plasmapause position appears on the nightside. In two cases, after SSC onsets, the plasmapause at noon and midnight was observed to retreat earthward approximately in one L-shell per hour. Similarly, for quiet periods, the plasmapause was found to expand at the same speed. This suggests that the time scales for the earthward and anti-earthward motion of the plasmapause are only a few hours. For the studies of the plasmapause position, it is, therefore, enough to consider the geomagnetic activity during the preceeding 4-6h.

4. The plasma density is clearly higher in the dayside trough than in the nightside trough, particularly during quiet intervals. On the dayside the density is usually a few electrons per $\mathrm{cm}^{3}$, whereas on the nightside it is $0.1-1 \mathrm{~cm}^{-3}$, where the low values are for low $K_{p}$ and the high values for high $K_{p}$. The difference is likely set by the ionosphere which provides higher refilling rates on the dayside. For disturbed conditions, the nightside trough can be filled with particles from additional sources, such as the plasmasphere (detached material) and the magnetotail (fast transport due to strong convection).

5. The auroral zone is characterized by a highly variable plasma density with a mixture of density cavities, at various scale sizes, not always connected to regions of (large) auroral electric fields or to the geomagnetic activity level. Cavities are observed but their locations, strengths, and sizes can vary. Some of them can last for more than two hours, enabling them to be observed by Polar over both hemispheres. Electric field perturbations do not necessarily correlate with the cavities: there 
are cavities with no electric field disturbances, and vice versa, auroral electric fields are observed with no clear cavitation. Furthermore, the occurrence of auroral cavities either at low or high altitudes does not correlate in any simple way with geomagnetic activity.

Acknowledgements. This work is supported under NASA grant NAG5-3182.

Topical Editor G. Chanteur thanks G. Marklund and V. Pierrard for their help in evaluating this paper.

\section{References}

Berchem, J. and Russell, C. T.: The thickness and velocity of the magnetopause current layer: ISEE 1 and 2 observations, J. Geophys. Res., 87, 2108-2114, 1982.

Carpenter, D. L. and Anderson, R. R.: An ISEE/Whistler model of equatorial electron density in the magnetosphere, J. Geophys. Res., 97, 1097-1108, 1992.

Elphic, R. C., Weiss, L. A., Thomsen, M. F., McComas, D. J., and Moldwin, M. B.: Evolution of plasmaspheric ions at geosynchronous orbit during times of high geomagnetic activity, Geophys. Res. Lett., 23, 2189-2191, 1996.

Gallagher, D. L., Craven, P. D., Comfort, R. H., and Moore, T. E.: On the azimuthal variation of core plasma in the equatorial magnetosphere, J. Geophys. Res., 100, 23 597-23 605, 1995.

Gallagher, D. L., Craven, P. D., and Comfort, R. H.: A simple model of magnetospheric trough total density, J. Geophys. Res., 103, 9293-9297, 1998.

Gallagher, D. L., Craven, P. D., and Comfort, R. H.: Global core plasma model, J. Geophys. Res., 105, 18 819-18 833, 2000.

Higel, B. and Lei, W.: Electron density and plasmapause characteristics at $6.6 R_{E}$ : a statistical study of the GEOS 2 relaxation sounder data, J. Geophys. Res., 89, 1583-1601, 1984.

Laakso, H. and Jarva, M.: Position and motion of the plasmapause, J. Atmos. Terr. Sol. Phys., 63, 1171-1178, 2001.

Laakso, H., Pfaff, R., and Janhunen, P.: Polar observations of electron density distribution in the Earths magnetosphere. 1. Statistical results, Ann. Geophysicae, this issue, 2002.

Lawrence, D. J., Thomsen, M. F., Borovsky, J. E., and McComas, D. J.: Measurements of early and late time plasmasphere refilling as observed from geosynchronous orbit, J. Geophys. Res., 104,
14 691-14 704, 1999.

Lemaire, J. F. and Gringauz, K. I.: The Earth's Plasmasphere, Cambridge Univ. Press, Cambridge, 1998.

Lindqvist, P.-A. and Marklund, G. T.: A statistical study of highaltitude electric fields measured on the Viking satellite, J. Geophys. Res., 95, 5867-5876, 1990.

Lyons L. R. and Williams, D. J.: Quantitative Aspects of Magnetospheric Physics, D. Reidel, Dordrecht, 1984.

Marklund, G. T., Blomberg, L. G., Falthamma, C.-G., Erlandson, R. E., and Potemra, T. A.: Signatures of the high-altitude polar cusp and dayside auroral regions as seen by the Viking electric field experiment, J. Geophys. Res., 95, 5767-5780, 1990.

Makela, J. S., Malkki, A., Koskinen, H., Boehm, M., Holback, B., and Eliasson, L.: Observations of mesoscale auroral plasma cavity crossings with the Freja satellite, J. Geophys. Res., 103, 9391-9404, 1998.

Moldwin, M. B.: Outer plasmaspheric plasma properties: what we know from satellite data, Space Sci. Rev., 80, 181-198, 1997.

Moldwin, M. B., Thomsen, M. F., Bame, S. J., McComas, D. J., and Moore, K. R.: An examination of the structure and dynamics of the outer plasmasphere using multiple geosynchronous satellites, J. Geophys. Res., 99, 11 475-11 481, 1994.

Newell, P. T. and Meng, C.-I.: Mapping the dayside ionosphere to the magnetosphere according to particle precipitation characteristics, Geophys. Res. Lett., 19, 609-612, 1992.

Palmroth, M., Laakso, H., and Pulkkinen, T.: Location of highaltitude cusp during steady solar wind conditions, J. Geophys. Res., 106, 21 109-21 122, 2001.

Park, C. G., Carpenter, D. L., and Wiggin, D. B.: Electron density in the plasmasphere: Whistler data on solar cycle, annual, and diurnal variations, J. Geophys. Res., 83, 3137, 1978.

Persoon, A. M., Gurnett, D. A., and Shawhan, S. D.: Polar cap electron densities from DE 1 plasma wave observations, J. Geophys. Res., 88, 10 123-10 136, 1983.

Persoon, A. M., Gurnett, D. A., Peterson, W. K., Waite, Jr., J. H., Burch, J. L., and Green, J. L.: Electron density depletions in the nightside auroral zone, J. Geophys. Res., 93, 1871-1895, 1988.

Rosenbauer, H., Grûnwaldt, H., Montgomery, M. D., Paschmann, G., and Sckopke, N.: Heos 2 observations in the distant polar magnetosphere: the plasma mantle, J. Geophys. Res., 80, 2723 2737, 1975.

Smith, M. F. and Lockwood, M.: Earth's magnetospheric cusps, Rev. Geophys., 34, 233-260, 1996. 